\title{
Extracción, pruebas de estabilidad y análisis químico preliminar de la fracción colorante obtenido a partir del exocarpo del fruto de Renealmia Alpinia
}

\author{
Extraction, stability testing and preliminary chemical analysis of the dye fraction \\ obtained from the exocarp of the fruit of Renealmia Alpinia
}

Paco Noriega Rivera*, Pablo Coba Santamaría, Joselito Naikiai y Juan Abad

Centro de Investigación y Valoración de la Biodiversidad CIVABI, Universidad Politécnica Salesiana, Quito, Ecuador.

* Autor para correspondencia: pnoriega@ups.edu.ec.

Artículo recibido el 30 de marzo de 20 I I.Aceptado, tras revisión el 20 de junio de 20 I I.

\begin{abstract}
Resumen
Del exocarpo del fruto comestible de la especie Renealmia Alpinia, proveniente de la Amazonía sur del Ecuador, mediante una extracción alcohólica y posterior evaporación del solvente, se obtuvo una fracción colorante de tonalidad púrpura con un rendimiento del 2,13\%.A dicha fracción se la sometió a diversas pruebas para analizar su estabilidad en variadas condiciones como: $\mathrm{pH}$, temperatura y su comportamiento con solventes de diversa polaridad. Para conocer anticipadamente su naturaleza química se efectuaron diversos ensayos entre los que destacamos: su espectro ultravioleta visible, su espectro infrarrojo, la cuantificación de antocianos totales y sus perfiles cromatográficos en HPLC a 520 nanómetros. Adicionalmente, se evaluó la toxicidad de la especie.
\end{abstract}

Palabras clave: Renealmia Alpinia, colorantes naturales, antocianinas.

\begin{abstract}
From the exocarp of the edible fruit Renealmia Alpinia, a natural species from the southern Ecuador Amazonia, we extract a purple dye.After an alcoholic extraction and subsequent evaporation of the solvent, we obtained a dye with a yield of $2.13 \%$. The dye was subject of various tests to analyze their stability to different conditions such as $\mathrm{pH}$, temperature and their behavior to solvents of different polarity. For preliminary chemical characterization ultraviolet, visible and infrared spectra were taken, as well as quantification of total anthocyanins and HPLC chromatographic profiles at 520 nanometers. Additionally we assessed the toxicity of the species.
\end{abstract}

Keywords: Renealmia Alpinia, natural dye, anthocyanins. 


\section{Introducción}

Las plantas del género Renealmia L.f Zingiberaceae, se encuentran ampliamente distribuidas a lo largo de los trópicos de América y África, con un total de 82 especies, de las cuales 62 se hallan en América (Mass \& Mass Van de Kamer, 200I). En Ecuador son cultivadas debido a sus frutos de naturaleza picante (Mass, 1976); crece en alturas comprendidas entre los 50 y $1.500 \mathrm{msm}$ (Maas \& Macía, 2005). Para el pueblo Shuar del Ecuador el fruto de la Renealmia alpinia constituye una importante fuente alimenticia, pero también de su exocarpo se extrae un colorante para pintar sus artesanías; el nombre tradicional que este pueblo da a la planta es Kumpia.

R.alpinia es una planta herbácea de $2 \mathrm{~m}$ de alto aproximadamente, hojas simples, alternas, sin estípulas, largo-lanceoladas, hasta $110 \mathrm{~cm}$ de longitud y $11 \mathrm{~cm}$ de ancho, nervadura paralela, vaina de la hoja abierta, con lígula. Inflorescencia racimosa, basal, $20-50 \mathrm{~cm}$ de longitud; brácteas de la inflorescencia rosado-rojizas, raquis pardo-rojizo; flor tubular, amarilla o rojiza. Fruto en cápsula, rojo cuando inmaduro, negro al madurar, ovoide, 3-4 cm de largo y I,5-2 cm de diámetro, posee numerosas semillas embebidas en una pulpa amarilla (Manuel J. Macía, 2003).

En los últimos años, el uso de colorantes de origen natural ha ganado gran importancia en el sector industrial, debido a la serie de observaciones que se han realizado a los colorantes sintéticos (Feakes \& Giusti, 2003). El grupo natural que reviste la mayor importancia dentro de los compuestos naturales coloreados son los flavonoides, no solo por presentar una gama amplia de diversas coloraciones, sino también porque últimamente se les atribuye un sinnúmero de características funcionales beneficiosas para la salud como: dilatador arterial, hepatoprotector, antioxidante, colerético, antifúngico, entre otras. Químicamente son sustancias fenólicas cuya característica principal es la de estar formadas por dos anillos bencénicos unidos por un puente de tres átomos de carbono C6-C3-C6 (Cartoya \& Reynaldo, 200I) (Figura I). Se dividen en: antocianinas, isoflavonas, auronas, flavonas, flavononas, flavonoles, chalconas, flavandioles y dihidroflavonoides; los cuales se diferencian entre sí, por los diversos grupos que se sustituyen alrededor del esqueleto básico.

Las antocianinas son metabolitos secundarios de gran interés debido a varios factores entre los que destacan: una amplia gama de diversas coloraciones como la azul, roja y morada (Rein y Maarit, 2002), una gran solubilidad en agua (Mozetic et al., 2002), potenciales beneficios a la salud humana (Giusti et al., 1999) y una inexistente toxicidad. Las antocianinas presen-

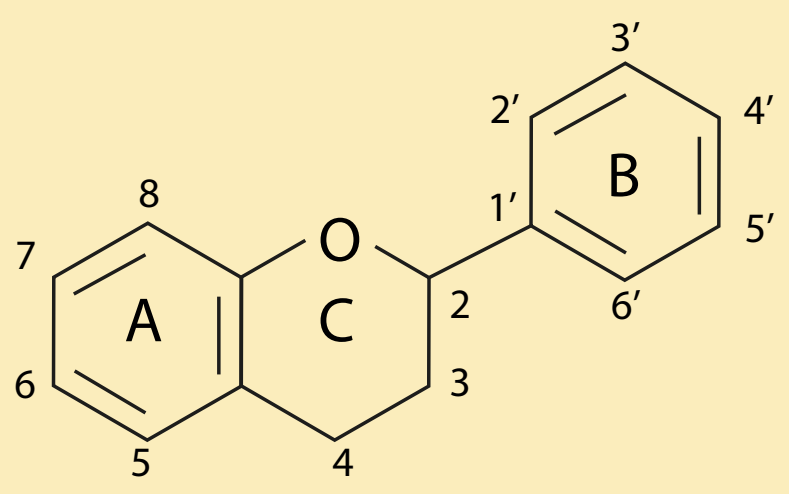

Figura 1. Estructura básica de los flavonoides

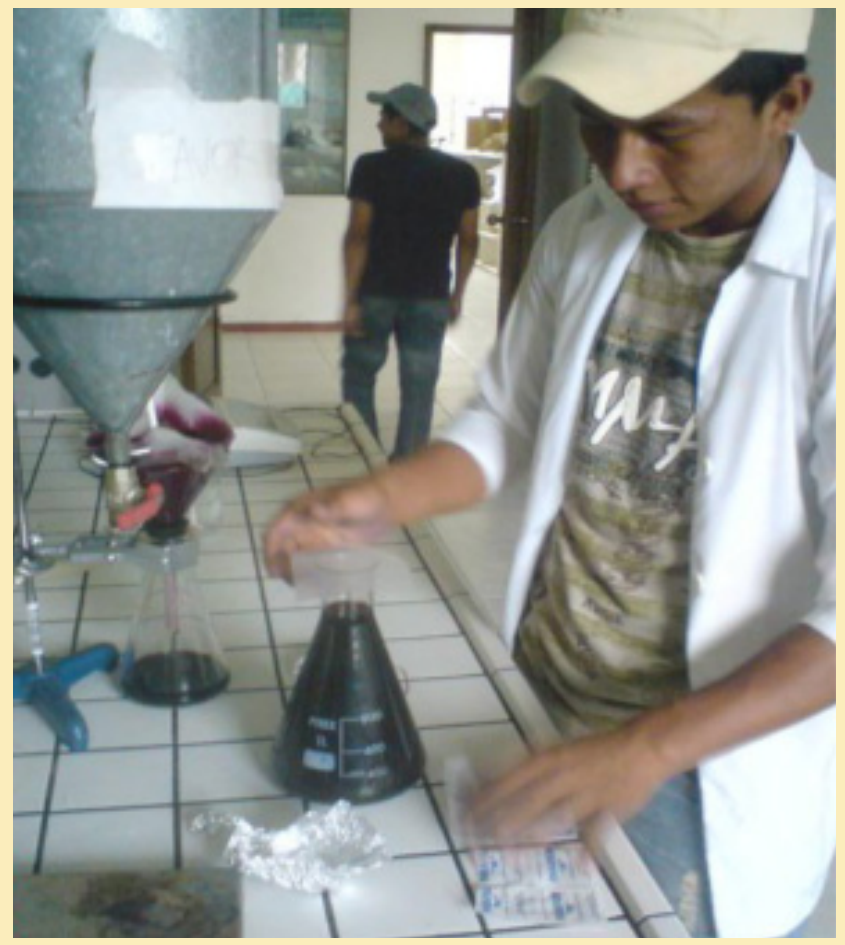

Figura 2. Proceso de extracción de la fracción colorante mediante percolación. Fuente: el autor.

tan espectros en el ultravioleta y visible en donde se evidencian dos bandas generales: la primera a longitudes de onda entre 270 a $280 \mathrm{~nm}$ y la segunda entre 465 y 560 nm (Navarro y Fabiola, 20 I0).

En la actualidad, la demanda de colorantes de naturaleza antociánica es muy alta, su producción se calcula entre 1.200 y I.300 toneladas anuales. Los requerimientos más importantes provienen de la industria alimenticia, debido a que en varios países de la Unión Europea, Sudeste Asiático y Latinoamérica su empleo no presenta restricciones (Ottersäater, 1999). 


\section{Materiales y métodos}

\section{I Extracción de la fracción colorante}

Para la extracción del colorante se empleó el método de percolación (ver Figura 2), utilizando como solvente una mezcla etanol-agua al $70 \%$. La cantidad de exocarpo fue de $2 \mathrm{~kg}$ para cada proceso de extracción. El extracto se recogió a las 48 horas y se procedió a evaporar el solvente en un rotavapor hasta obtener una masa pastosa, la cual se recogió en un recipiente amplio de vidrio. Posteriormente, se evaporó todo el solvente en una estufa y se determinó el rendimiento.

\subsection{Pruebas de estabilidad de la fracción co- lorante}

\subsection{Pruebas de comportamiento a diferentes $\mathrm{pH}$}

Se evaluó el comportamiento en valores de $\mathrm{pH}$ de, 4, 6, 8 y 12 empleando soluciones diluidas de $\mathrm{HCl}$ y $\mathrm{NaOH}$.

\subsubsection{Pruebas de comportamiento a diferen- tes temperaturas}

Los ensayos para determinar el comportamiento de la fracción colorante ante la temperatura se hicieron como se describe en la Tabla I. Se empleó una solución acuosa del colorante a una concentración del $0,5 \%$.

Tabla I. Condiciones para evaluar el efecto de la temperatura

\begin{tabular}{cc}
\hline Temperatura & Tiempo (minutos) \\
\hline $86^{\circ} \mathrm{C}$ & 10 \\
$86^{\circ} \mathrm{C}$ & 60 \\
$100^{\circ} \mathrm{C}$ & 30 \\
$5^{\circ} \mathrm{C}$ & 30 \\
\hline
\end{tabular}

\subsubsection{Pruebas de solubilidad}

Para analizar la solubilidad del colorante se emplearon varios solventes, tanto de naturaleza polar como apolar. Los solventes usados fueron: agua, solución de ácido cítrico al $10 \%$, metanol, glicerina, aceite de palma y hexano.

\subsection{Ensayos físicos y químicos preliminares}

\subsection{Espectro visible de la fracción colorante}

Para evaluar la longitud de onda máxima de absorción se usó un espectrofotómetro UV/VIS marca Shimadzu, modelo UV 1240; el rango para obtener el espectro fue entre 400 y $700 \mathrm{~nm}$. La solución empleada tuvo una concentración de 1.000 ppm.

\subsubsection{Espectro infrarrojo de de la fracción colorante}

Para obtener el espectro infrarrojo del colorante se uso un FT-IR, marca Perkin Elmer, modelo Spectrum BX; la muestra se analizó directamente fabricando una pastilla con bromuro de potasio.

\subsubsection{Cuantificación de las antocianinas totales}

Para la cuantificación de las antocianinas totales se empleó el método de $\mathrm{pH}$ diferencial descrito por (Wrolstad, 1993). Dado que los cálculos fueron hechos sobre el extracto colorante seco se procedió a cuantificar el porcentaje peso/peso de antocianinas, siguiendo la metodología de la investigación realizada por (Del Carpio Jiménez et al., 2009), dicho procedimiento calcula la absorbancia del colorante de manera diferencial a $\mathrm{pH} \mathrm{I}, 0$ y $\mathrm{pH} 4,5$ y considerando su absorbancia máxima a $520 \mathrm{~nm}$ y la absorbancia a $700 \mathrm{~nm}$.

La absorbancia total se obtiene usando la siguiente expresión:

$$
\mathrm{A}=(\text { Amax-A700)pH1-(Amax-A700)pH4,5 }
$$

El contenido total de antocianinas monoméricas expresadas como cianidina-3-glucósido se calcula con la siguiente expresión:

Contenido de antocianinas

$$
(\mathrm{p} / \mathrm{p})=(\mathrm{A} \times \mathrm{MW} \times \mathrm{DF} \times \mathrm{V} \times \mathrm{Wt}) /(\xi \times \mathrm{L})(2)
$$

Donde:

$\mathrm{A}=$ Absorbancia total.

MW= peso molecular de la cianidina-3-glucósido= 449,6 g/L.

$\mathrm{DF}=$ Factor de dilución.

$\mathrm{V}=$ volumen en $\mathrm{mL}$.

$\mathrm{Wt}=$ peso de la muestra en $\mathrm{mg}$

$\xi=\quad$ absortividad molar de la cianidina-3-glucósido= 26900.

$\mathrm{L}=$ grosor de la cubeta $=\mathrm{Icm}$. 


\subsubsection{Perfil HPLC de la fracción colorante}

Antes de inyectar la muestra, se procedió a hidrolizarla, para esto se realizó una hidrólisis empleando $\mathrm{HCL}$ $4 \mathrm{~N}$ con I hora de reflujo, como se describe en los protocolos (Wrolstand et al., 200 I).

Para el análisis se empleo un equipo de cromatografía líquida de alta resolución HPLC marca Varian modelo ProStar 320, una columna Cl 8 marca Varian de $150 \times 4,6 \mathrm{~mm}$, con un diámetro de partícula de 5 um. El flujo fue de $0,1 \mathrm{~mL} / \mathrm{min}$; la fase móvil A constituida por una solución de ácido acético al $10 \%$, la fase móvil $B$ acetonitrilo; la gradiente empleada fue 0-6 minutos $\% \mathrm{~A}$ y $0 \% \mathrm{~B}, 6-20$ minutos $90 \% \mathrm{~A}$ y $10 \% \mathrm{~B}, 20-80$ minutos $95 \%$ A y $5 \% \mathrm{~B}$. La longitud de onda de análisis fue de $520 \mathrm{~nm}$.

\subsection{Evaluación de la toxicidad de Renealmia alpinia}

La evaluación de la toxicidad se la hizo empleando el método de la artemia salina detallado por (Sharapin,
1995), para una muestra que contenía diversas partes de la planta.

\section{Resultados y discusión}

\section{I Extracción de la fracción colorante}

Se realizaron varios procesos de extracción obteniéndose un rendimiento medio de $2,13 \%$, con un promedio de $43 \mathrm{~g}$ de fracción colorante por cada $2 \mathrm{~kg}$ de exocarpo usado.

\subsection{Pruebas de estabilidad de la fracción co- lorante}

\subsection{Pruebas de comportamiento a diferente $\mathrm{pH}$}

La evaluación del comportamiento a diversos $\mathrm{pH}$ dio como resultado comportamientos disimiles. Tabla 2 y Figura 2.

Tabla 2. Comportamiento del colorante a diversos $\mathrm{pH}$

\begin{tabular}{ll}
\hline $\mathbf{p H}$ & Observaciones \\
\hline $\mathbf{4}$ & Coloración rojo oscuro. \\
$\mathbf{6}$ & Coloración rojizo translucido. \\
$\mathbf{8}$ & Color morado (uva) translúcido. \\
$\mathbf{1 2}$ & $\begin{array}{l}\text { Los primeros tres minutos dio una coloración morado (uva) no traslucido, } \\
\text { luego cambia a un color ámbar translucido. }\end{array}$ \\
\hline
\end{tabular}

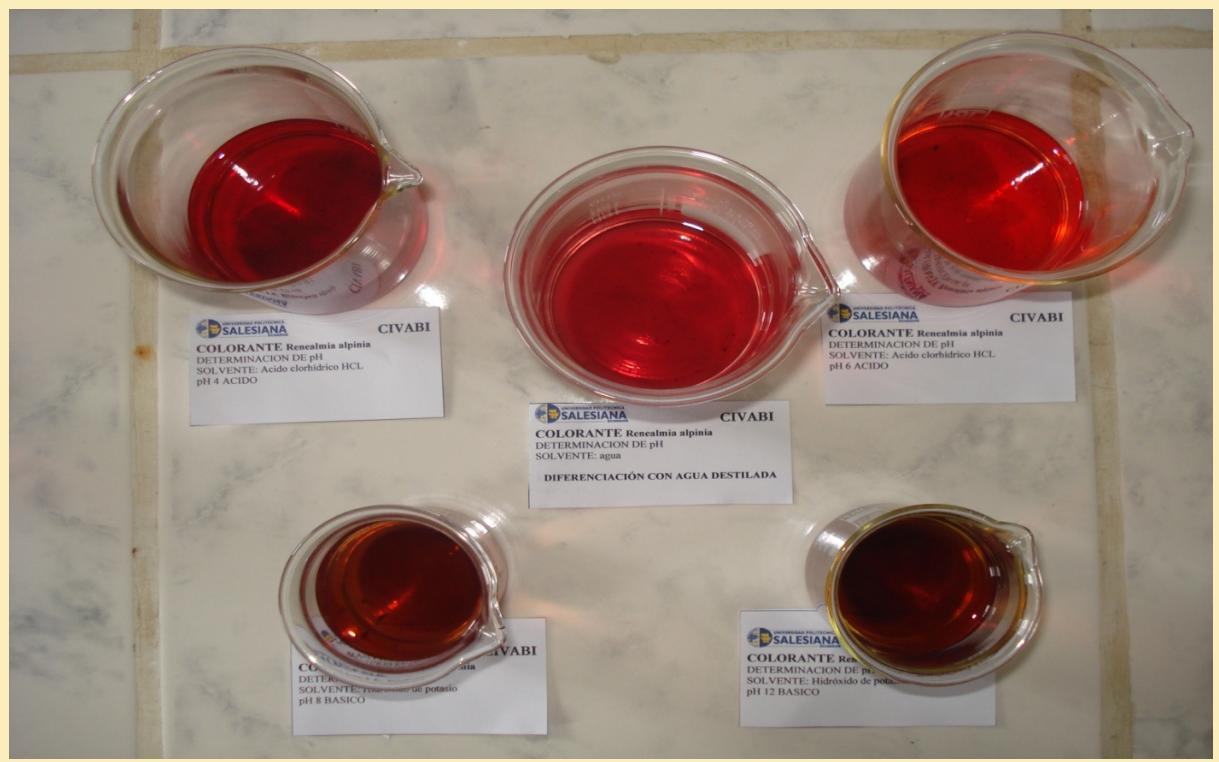

Figura 2. Comportamiento del colorante a diversos $\mathrm{pH}$ : fila superior. $\mathrm{pH} 4,7$ y 6; fila inferior $\mathrm{pH} 8$ y 12. Fuente: el autor. 


\subsubsection{Pruebas de comportamiento a diferentes tempe- raturas}

Los ensayos para el comportamiento a diferentes temperaturas arrojaron los siguientes resultados (Tabla 3).

Tabla 3. Resultados del comportamiento del colorante a diferentes temperaturas

\begin{tabular}{ccl}
\hline $\begin{array}{c}\text { Temperatura } \\
\left({ }^{\circ} \mathbf{C}\right)\end{array}$ & $\begin{array}{c}\text { Tiempo } \\
(\mathbf{m i n})\end{array}$ & Observaciones \\
\hline $86^{\circ} \mathrm{C}$ & 10 minutos & $\begin{array}{l}\text { Color rojizo transpa- } \\
\text { rente no cambia al ca- } \\
\text { lentarse. } \\
\text { Color rojizo, no existe } \\
\text { cambios de coloración. } \\
86^{\circ} \mathrm{C}\end{array}$ \\
$100^{\circ} \mathrm{C}$ & 30 minutos & $\begin{array}{l}\text { No existen cambios de } \\
\text { coloración. }\end{array}$ \\
$5^{\circ} \mathrm{C}$ & 30 minutos & $\begin{array}{l}\text { No existen cambios de } \\
\text { coloración. }\end{array}$ \\
\hline
\end{tabular}

\subsubsection{Pruebas de solubilidad}

Las pruebas de solubilidad del colorante dieron como resultado lo descrito en la (Tabla 4 y Figura 3).

\subsection{Ensayos físicos y químicos preliminares}

\subsection{Espectro visible de la fracción colorante}

Se obtuvo un espectro de una solución de 1.000 ppm del colorante, en donde la longitud de onda de máxima absorbancia se produce a los $537 \mathrm{~nm}$.

\subsubsection{Espectro infrarrojo de la fracción colorante}

Se obtuvo el espectro infrarrojo (ver Figura 4), en donde se pueden observar varias señales de grupos funcionales entre las que destacan: un pico a 3402 $\mathrm{cm}^{-1}(\mathrm{OH})$, un pico a $1.609 \mathrm{~cm}^{-1}$ (grupo carbonilo), los cuales son característicos de los compuestos de tipo flavínico.

Tabla 4. Pruebas de solubilidad

$\mathrm{H}_{2} \mathrm{O}$

Acido Cítrico $10 \%$

Metanol

Glicerina

Aceite de palma

Hexano
Se disuelve fácilmente tomando una coloración rojiza intensa traslucida.

Se disuelve fácilmente. Toma una coloración rojiza y translucida. Se potencializa la coloración.

Nos se disuelve fácilmente, Resulta un color morado oscuro (uva) y en su contextura se observa pequeñas partículas del colorante. Después de $1 / 2$ hora cambia de color a plomizo.

Se disuelve mientras se lo agita, da una coloración morado con translucido rojizo.

Casi no se mezcla, quedan grumos del colorante, toma un color púrpura. 


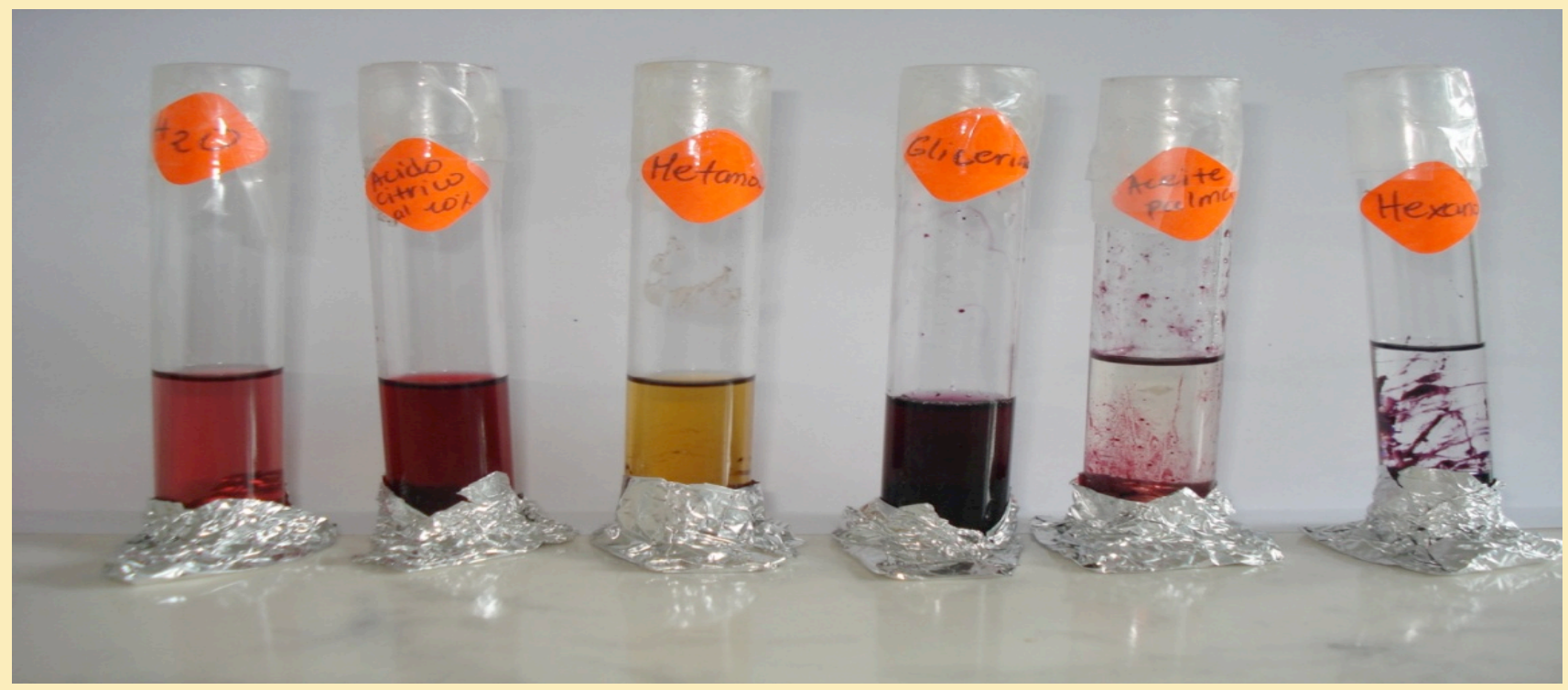

Figura 3. Pruebas de solubilidad: agua, ácido cítrico al 10\%, metanol, glicerina, aceite de palma y hexano. Fuente: el autor.

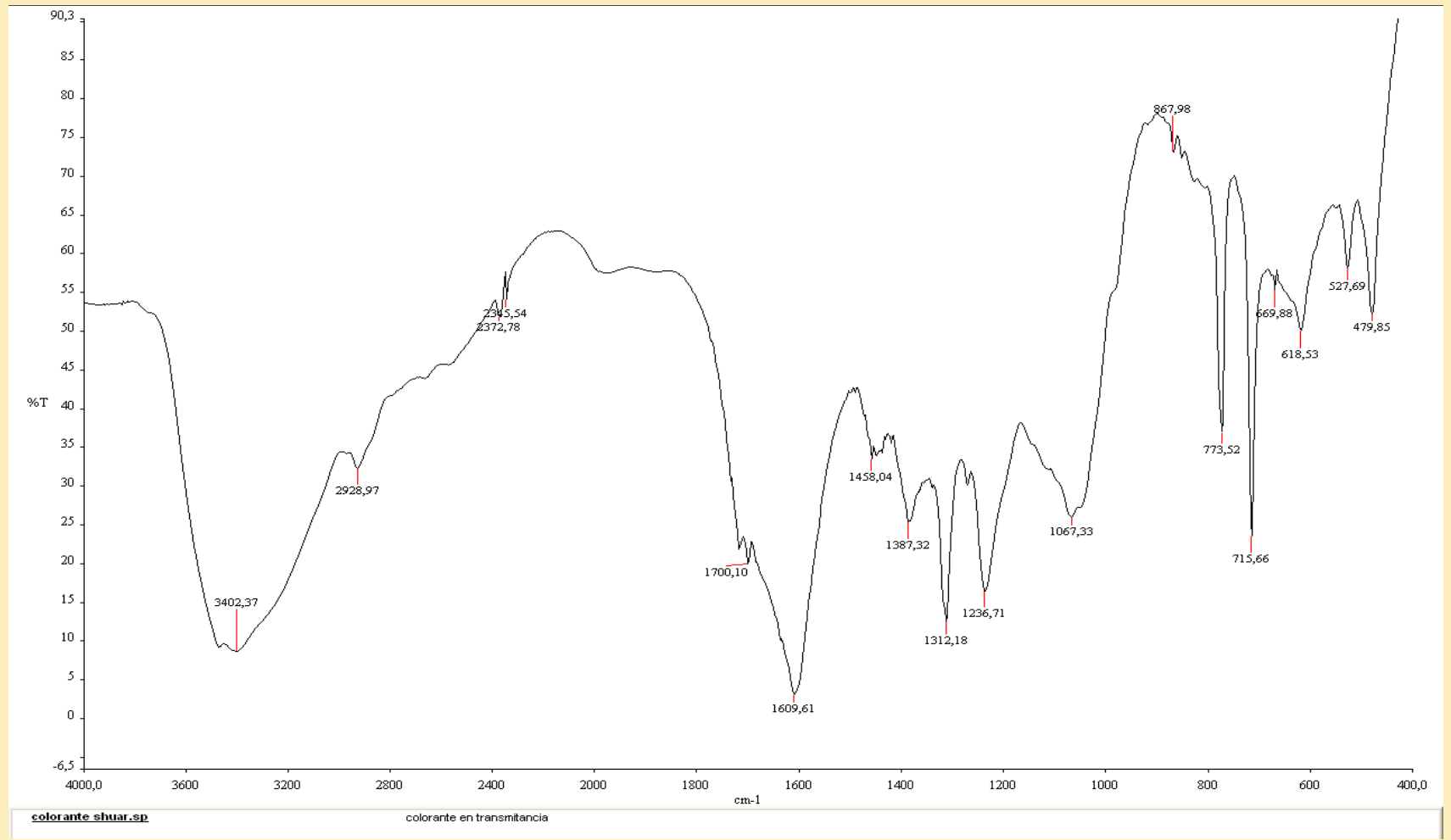

Figura 4. Espectro infrarrojo del colorante

\subsubsection{Cuantificación de antocianos totales}

El valor de absorbancia total para una solución de I.000 ppm del colorante disuelto en agua fue de 0,167.

Aplicando la fórmula para calcular el porcentaje $(p / p)$ de antocianos totales expresados como cianidina-3-glucósido, tenemos que en el colorante obtenido por extracción etanólica del exocarpo de Renealmia alpinia la concentración de antocianos es del $27,9 \%$. 


\subsubsection{Perfil HPLC de la fracción colorante a $520 \mathrm{~nm}$}

El análisis del perfil de antocianos a $520 \mathrm{~nm}$ (ver Figura 5), nos indica la presencia de tres moléculas claramente definidas, las cuales aparecen de la siguiente manera: un primer pico pequeño a los I5,44 minutos; un segundo a los 17,39 minutos, que en este caso es el compuesto mayoritario; $y$, un tercero a los 19,01 minutos.

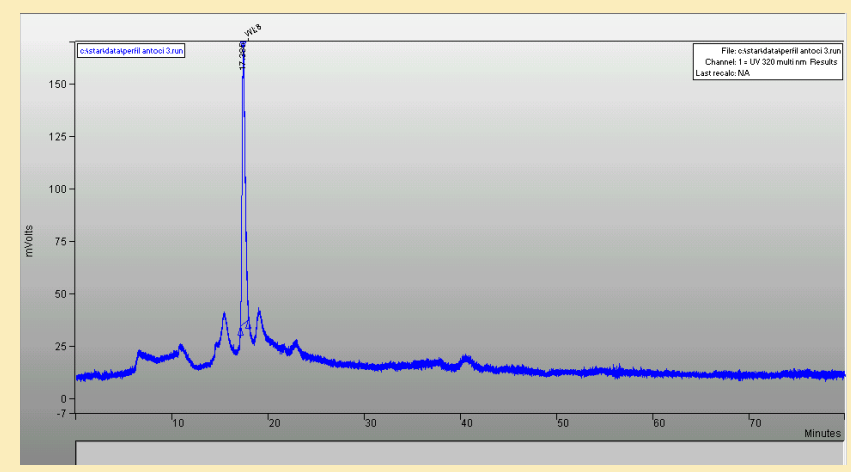

Figura 5. Perfil de componentes de naturaleza antocíanica obtenidos a una longitud de onda de $520 \mathrm{~nm}$

\subsection{Evaluación de la toxicidad del fruto de Renealmia alpinia}

Los resultados de la evaluación de toxicidad efectuados en la especie vegetal Renealmia alpinia (Tabla 5) denotan su total inocuidad y su uso seguro como colorante natural.

\section{Conclusiones}

La fracción colorante tiene un alto potencial para convertirse en una fuente de uso alternativo natural para las industrias alimenticia y cosmética. Su alta solubilidad en solventes polares, su tolerancia a altas temperaturas, y su inocuidad sustentan esta afirmación. El rendimiento resulta interesante, sobre todo, al considerar que su extracción proviene de un subproducto de desecho como es el exocarpo. El proceso de extracción resulta muy económico y amigable con el ambiente, pues emplea un solvente compuesto de etanol y agua. Se observa un comportamiento estable en medios neutro y ácido, y un

Tabla 5. Evaluación de la toxicidad de Renealmia alpinia.

\begin{tabular}{|c|c|c|c|c|c|c|c|c|c|c|c|c|c|}
\hline \multicolumn{2}{|c|}{$\begin{array}{c}\text { Fecha de ensayo } \\
\text { del } 16 / 05 / 08 \\
\text { al } 04 / 06 / 08\end{array}$} & \multicolumn{2}{|c|}{$\begin{array}{l}\text { Primera } \\
\text { repetición }\end{array}$} & \multicolumn{2}{|c|}{$\begin{array}{l}\text { Segunda } \\
\text { repetición }\end{array}$} & \multicolumn{2}{|c|}{$\begin{array}{l}\text { Tercera } \\
\text { repetición }\end{array}$} & \multicolumn{3}{|c|}{$\begin{array}{l}\text { DL } 50 \text { ppm } \\
>1000 \\
1000-100 \\
100-10\end{array}$} & \multicolumn{3}{|c|}{$\begin{array}{c}\text { Toxicidad } \\
\text { No tóxica } \\
\text { Medianamente tóxica } \\
\text { Tóxica } \\
\text { Muy tóxica }\end{array}$} \\
\hline 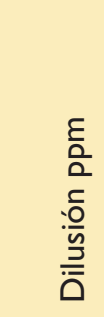 & 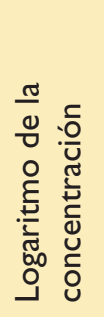 & 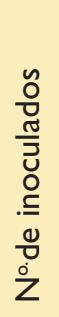 & 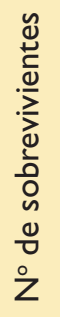 & 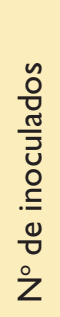 & 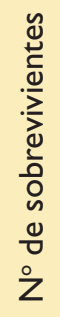 & 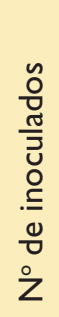 & 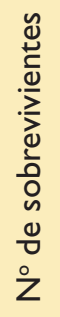 & $\begin{array}{l}\overline{\frac{\pi}{\sigma}} \\
\frac{\tilde{\omega}}{\Sigma}\end{array}$ & $\begin{array}{l}\gtrsim \\
\frac{\pi}{\pi} \\
\frac{\pi}{2}\end{array}$ & 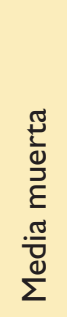 & 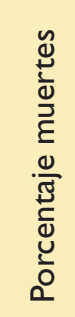 & $\begin{array}{l}\text { 유 } \\
\text { II } \\
\text { > }\end{array}$ & ㅇํํ \\
\hline 1.000 & 3 & 10 & 0 & 10 & 6 & 10 & 0 & 10 & 2 & 8 & 0 & & 50.816 \\
\hline 500 & 2,699 & 10 & 7 & 10 & 7 & 10 & 7 & 10 & 10 & 3 & 0 & 0 & \\
\hline 100 & 2 & 10 & 10 & 10 & 9 & 10 & 10 & 10 & 9,7 & 0,3 & 0 & & \\
\hline 50 & 1,699 & 10 & 10 & 10 & 10 & 10 & 10 & 10 & 10 & 0,0 & 0 & & \\
\hline 10 & I & 10 & 10 & 10 & 10 & 10 & 10 & 10 & 10 & 0,0 & 0 & 3 & 1.000 \\
\hline
\end{tabular}


cambio de coloración al entrar en contacto con medios alcalinos.

Los estudios químicos preliminares arrojan valiosa información. Hay una presencia alta de compuestos fenólicos de naturaleza antociánica, confirmada en los diversos estudios espectrofotométricos en el infrarrojo y en el visible. El perfil cromatográfico a 520 $\mathrm{nm}$ demuestra la presencia preponderante de una antocianina, cuya naturaleza química posteriormente podría se elucidada por métodos como el NMR y la espectrometría de masas.

Si bien es cierto, se concluye que se trata de una variedad no tóxica. Se recomienda realizar estudios que evalúen la misma en el colorante puro.

\section{Referencias}

Cartoya O. e Inés Reynaldo. 200I. Flavonoides: Características Químicas y Aplicaciones. Cultivos Tropicales. 200I. Vol. 22. № 2: 5-I4.

Del Carpio Jimenes Carla, Serrano Flores Carlos y Giusti Mónica. 2009. Caracterización de las antocianinas de los frutos de Berberis boliviana Lechler. Rev. Soc. Quím. Perú 75(I)2009.

Feakes D.M. y M. M. Giusti. 2003. Feasibility of the use of acylated anthocyanins to color low acid dairy products. 2003 IFT Annual Meeting-Chicago. 2003.

Giusti M.M, Rodriguez-Saona, L y R. E. Wrolstand. 1999. Molar adsorptivity and color characteristics of acylated and non acylated pelargonidin-based anthocyanins, J. Agric. Food Chem 47: 4631-4637, 1999a.

Macía J. Manuel. 2003. Renealmia alpinia (Rottb.) Maas (Zingiberaceae): planta comestible de la Sierra Norte de Puebla (México). Anales Jard. Bot. Madrid 60(I): |83-|87.

Mass, P. J. M. 1976. Zingiberaceae, en: G. Harling \& B Sparre (eds.), Flora of Ecuador 6: I-50, 1976.
Mass, P.J.M. 200I. Zingiberaceae Lindl, en:W.D. Stevens, C. Ulloa-Ulloa, A Pool \& O.M. Montiel (eds.), Flora de Nicaragua. Monographs in Systematic Botany from the Missouri Botanical Garden 85(3): 2549-2554. 200 I.

Maas H., Macia M.J. 2005. Zingiberaceae 577-580, en: Steyermark J. A., Berry P. E., Holst B. K. (eds.) Flora of the Venezuelan Guayana. 2005.

Mozetic Branka, Trebse Polanca, Hribar Janez. 2002. Determination and Quantitation of anthocyanins and hydroxycinnamics acids in different cultivars of sweet cherries (Prunus avium L) from Nova Gorica región (Slovenia). J. Food Technol. Biothecnol, 40(3) 207-2I2 2002.

Navarro. G \& R. Fabiola. 2010. Comprobación del efecto cicratizante de Peperomia scutellaefolia R. et P., aspectos etnofarmacológicos, botánicos y estudio químico. Tesis UNMSM 2010.

Ottersäater, G. 1999. Coloring of Food, Drugs and Cosmetics. New York, N.Y.: Marcel Dekker, Inc.; 1999.

Rein, Maarit. 2002. Copigmentation reactions and color stability of berry anthocyanins (Academic Dissertation) EKT series I53I. University of Helsinki. Departmen of Applied Chemistry and Microbiology. 2005.

Sharapin Nicolai. 1995. Fundamentos de tecnología de productos fitoterapéuticos. Editor Roberto Pinzon. 1995.

Wrolstad Ronald, E. 1993. Colour and Pigment Analyses in Fruit Productos. Agricultural Experiment Station Oregon State University Station Bulletin 624. 1993.

Wrolstad Ronald, E. y E. Acree Terry. Current protocols in food analytical chemistry, $\mathrm{Vol} \mathrm{I}$; Jonh Wiley and Sons Inc, F I.I.I-FI.3.9.200I. 